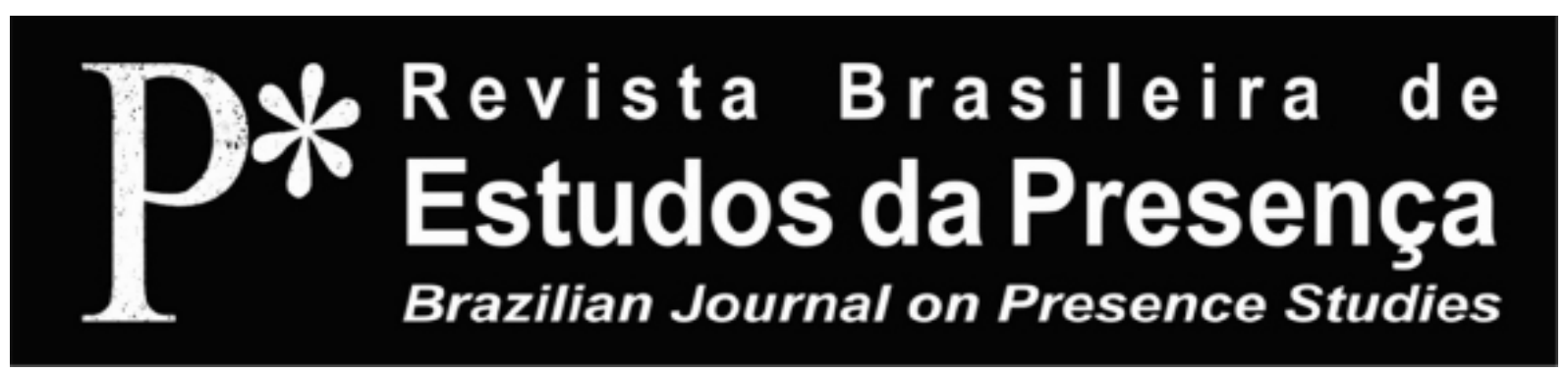

E-ISSN 2237-2660

\title{
Du Terrain Ethnographique à la Dramaturgie: une enquête théâtrale sur les afro-brésiliens du Togo, aujourd'hui
}

\author{
Bernard Müller \\ École des Hautes Études en Sciences Sociales - Paris, France
}

RÉSUMÉ - Du Terrain Ethnographique à la Dramaturgie: une enquête sur les afrobrésiliens du Togo, aujourd'hui - En associant les méthodes de l'anthropologie culturelle à celles du théâtre, nous envisageons dans cet article le processus de création artistique comme un terrain ethnographique. La démarche proposée vise à inverser la posture habituelle du chercheur qui conçoit sa position d'observation comme étant située à l'extérieur de l'objet étudié. En met littéralement en œuvre son terrain, le chercheur crée avec les enquêtés une situation qui permet de faire émerger une forme de connaissance anthropologique, sa nature et sa place parmi les sciences posant question.

Mots-clés: Ethnologie. Dramaturgie. Diaspora. Afro-Brésiliens. Théâtre.

ABSTRACT - From Ethnographic Work to Dramaturgy: a theatrical research on the afro-brazilians of Togo, today - By combining methods from cultural anthropology to the methods of theatre, this article considers the process of artistic creation as ethnographic field work. The proposed approach aims to reverse the usual position of the researcher who regards his/her position of observer as external to the object of study. By placing his/her own field literally in the work, the researcher creates, along with the respondents, a situation which enables the emergence of a form of anthropological knowledge that questions its nature and place among the sciences.

Keywords: Ethnology. Dramaturgy. Diaspora. African-Brazilian. Theatre.

RESUMO - Do Trabalho Etnográfico à Dramaturgia: uma pesquisa teatral sobre os afro-brasileiros do Togo, atualmente ${ }^{1}$ - Ao combinar os métodos da antropologia cultural aos do teatro, este artigo considera o processo de criação artística como um campo de trabalho etnográfico. A abordagem proposta visa inverter a postura habitual do pesquisador, considerado como um observador externo ao objeto estudado. Ao colocar literalmente o seu campo à obra, o pesquisador cria juntamente com os entrevistados uma situaçáo que possibilita o surgimento de uma forma de conhecimento antropológico, que questiona sua natureza e seu lugar entre as ciências.

Palavras-chave: Etnologia. Dramaturgia. Diáspora. Afro-brasileiros. Teatro. 


\section{Introduction: une Nuit de Décembre ${ }^{2}$}

Dans les environs de la ville côtière d'Aného au Togo, j'assistais dans la nuit du 14 au 15 décembre 2011 à une étonnante cérémonie. Dans la cour du palais du roi de Glidji, à proximité du sanctuaire des 42 divinités (vaudous) du peuple Guin-Mina, se déroulait sous une grande tente en toile verte, une sortie de deuil peu habituelle, fermant ainsi un cycle de cérémonies destinées à rendre un dernier hommage aux grands prêtres décédés en esclavage. Ce rituel de fin de deuil (appelé tchessi koko en langue mina-guin, qui est la langue parlée dans cette région) constituait la dernière étape d'un long processus qui avait débuté deux ans plus tôt, lors d'une cérémonie inaugurale organisée autour des restes de prêtres morts en exil au Brésil, rapatriés dans leur terre natale, du moins - me dit-on - à partir de sable et de poussières prélevés récemment dans divers cimetières des alentours de Salvadorde Bahia.

Les restes de ces prêtres africains avaient été rapportés du Brésil par la compagnie du Balé Folcorico de Salvador de Bahia qui participe depuis l'origine au Festival des Divinités Noires et dont certains membres appartiennent à un terreiro ${ }^{3}$ de candomblé de Salvador. Ce festival, résolument placé sous le signe d'une rencontre à la fois religieuse et artistique, a été lancé par Yves Têtê Wilson-Bahoun, un notaire de la capitale, originaire d'Aného, ville côtière voisine de Glidji, ancienne capitale du Togo allemand. Celui-ci rappelle souvent avec insistance et émotion que "le festival ne serait pas ce qu'il est sans la participation des Afro-Brésiliens" (Communication personnelle, décembre 2011). Selon Maître Wilson, le Brésil fait partie de l'histoire du Togo, de la même manière que la culture des peuples du Togo se retrouve dans celle du Brésil contemporain. Il rappelle souvent que les "Afro-Brésiliens sont chez eux ici comme l'étaient déjà leurs ancêtres revenus du Brésil au siècle dernier", se référant ici à ceux qu'on appelle les Agoudas (Guran, 2010) au Bénin et au Togo, les Amaros au Nigeria ou encore les Tabon (Amos; Ayesu, 2002) au Ghana et qui désignent les descendants d'esclaves originaires de la côte du golfe du Bénin et revenus libres du Brésil. De qui s’agit? Qui sont donc ces Brésiliens d'Afrique?

On sait en effet sans plus de précision statistique qu'entre 1793 et 1880 plusieurs milliers d'esclaves affranchis sont retournés d'Amérique vers la terre de leurs parents ou grands-parents. Il s'agit principalement des retornados, esclaves bannis du Brésil pour avoir 
participé aux révoltes qui secouèrent notamment Salvador de Bahia en 1805 , en 1822 puis en 1835. A ces exilés paradoxaux (on les expulse certes, mais on les renvoie chez eux) se rajoutèrent d'autres migrants à l'instar des returnees, parfois confondus avec les saros, composés de déserteurs de l'armée indépendantiste américaine ayant rejoint la colonie britannique du Canada en échange de leur liberté, des marroons de Jamaïque (reconquise en 1796) déportés en Nouvelle-Ecosse avant de repartir vers l'Afrique - accueillis notamment en Sierra Leone (Huber, 2004), puis au Liberia. A ces revenants d'Amérique s'ajouta encore un nombre croissant de recaptives. Ce sont les captifs embarqués puis rapidement libérés le long des côtes par les croiseurs britanniques, avant d'être débarqués également à Freetown ou Monrovia, pour parfois retourner dans leur région d'origine sans jamais avoir traversé l'océan. Enfin, il ne faut pas omettre les captifs enlevés à l'intérieur des terres qui furent déplacés sur la côte sans jamais embarquer, comme par exemple la population qui fonda le village d'Atoeta, essentiellement constituée d'une cargaison d'esclaves jamais partie. De nos jours encore, les habitants du village d'Atoeta savent qu'ils sont les descendants d'esclaves d'origine Fon, Nago, Haoussa, Kabyè, Tchamba, Moba, Tem, Nawdeba, Gourma, Konkomba, Mossi. A côté du nom qui rappelle cette origine, bon nombre d'entre eux portent aujourd'hui encore les noms à consonance portugaise de maîtres qu'ils n'ont jamais eus mais auxquels ils auraient dû être vendus, étrangement.

Cette histoire a fait l'objet de nombreuses recherches, en particulier à partir des archives brésiliennes et des récits des missionnaires rédigés tout au cours du XIX $\mathrm{X}^{\mathrm{e}}$ siècle. Par contre, peu de travaux ont été menés sur une période qui commence avec le protectorat français et qui s'étend jusqu'à aujourd'hui.

\section{Les Indices d'une Renaissance Culturelle}

Certains signes porteraient pourtant aujourd'hui à croire que l'on assiste à la reformation de ce groupe social, moment embryonnaire qui fait de nous les participants privilégiés d'une invention culturelle. Aujourd'hui, de Lagos (Soumonni, 2005) à Accra, en passant par Badagry, Porto-Novo, Cotonou, Ouidah (Law, 2004), Aného, Agoué, Lomé, Aflao, Keta, Anloga ou Tema, il est à nouveau de bon ton d'afficher ses origines brésiliennes et de rappeler que si l'on est d'ici, on est aussi d'ailleurs. 
Cet apparent regain s'exprime notamment par une valorisation des différences/spécificités brésiliennes dans les manifestations culturelles se déroulant sur la côte du golfe de Guinée, plus encore au Bénin qu'au Togo. Les fêtes traditionnelles dans les villes où les Brésiliens sont nombreux connaissent une publicité inédite: le carnaval gagne tous les quartiers de la ville de Ouidah et les bourinha (ou encore bouryan, inspirées du bumba-meu-boi brésilien) ${ }^{4}$ ont à nouveau pignon sur rue. Leurs pratiques religieuses bénéficient d'un intérêt nouveau: les cultes vaudous liés à la traite négrière (comme Mama Tchamba, Gorovodou, Mami Wata ou encore Hwla), les cultes luso-brésiliens des saints catholiques rapportés du Brésil, ou encore la forme d'Islam rapportée elle aussi du Brésil. Les événements culturels qui affichent leur intention de contribuer au rapprochement de l'Afrique de l'ouest et du Brésil se multiplient, à l'instar du Festival des Divinités Noires (mentionné ci-dessus) qui a tenu décembre

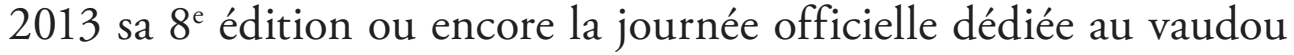
qui a lieu tous les 10 janvier au Bénin, et dont Ouidah, éminent port négrier, est l'épicentre. Logiquement, un tourisme thématique proposant un retour aux sources à ses participants se développe pour faire découvrir aux Brésiliens cette histoire commune et lui redonner un sens nouveau et moderne.

Cette réémergence culturelle, si elle est avérée, pourrait aussi être liée à une dynamique économique incontestablement marquée au cours de la dernière décennie par le développement des relations Sud/Sud, notamment avec le Brésil, l'Inde et la Chine, à un moment où les Etats africains du golfe de Guinée connaissent une croissance économique inconnue jusqu'alors (Le FMI a constaté une croissance d'environ 7\% en 2012 dans les pays de l'UEMOA et d'environ 5\% au Brésil). Cette causalité économique dans l'explication du mouvement étudié est toutefois à prendre avec précaution. Récemment (mars 2013), une ligne aérienne directe reliant 3 fois par semaine Lomé à Rio ou Sao Paolo a été ouverte par l'entreprenante Ethiopian Airlines.

Qui sont les acteurs de ce mouvement culturel et quels en sont les enjeux économiques, politiques ou culturels?

Comment rendre compte de ce travail culturel en cours? 


\section{Une Enquête Théâtrale}

Pour répondre à ces questions, il me parut intéressant de concevoir un mode de recherche qui associe ethnologie et théâtre, en imbriquant ainsi la construction de l'objet de recherche à un processus de création artistique.

Ce choix, inspiré par la démarche exposée par Johannes Fabian (1990), résulte à la fois d'une préoccupation épistémologique quant au statut de la connaissance anthropologique et de son rapport avec l'art, la littérature et plus spécifiquement l'écriture dramatique ainsi que d'un concours de circonstances. Dans l'idée de dépasser le relativisme absolu de la critique postmoderne et le simplisme réducteur des stratégies d'écriture (voir Clifford, 1986), dans l'espoir de déconstruire les relations de pouvoir qui sont à l'œuvre dans les processus modernes de production de savoir, en particulier en ethnographie ou en anthropologie culturelle.

Un concours de circonstances fit qu'en 2004, au cours d'une discussion avec Kangni Alem, le choix de mon thème de recherche s'est porté sur le roman qu'il était alors en train d'écrire (cet ouvrage paraîtra en 2009 aux éditions Lattès sous le titre d'Esclaves et il a été traduit en portugais et est paru aux éditions Pallas en 2011 sous le titre d'Escravos). Il s'agit d'une fiction historique dont le personnage central est emblématique du parcours des Afro-Brésiliens, capturés puis déportés au Brésil où ils deviendront des esclaves, avant de revenir libres une à deux générations plus tard sur une terre dont ils savaient qu'elle était celle de leurs ancêtres. Ce périple offre la trame d'une narration au cours de laquelle la position sociale des protagonistes se modifie sans cesse, pour passer d'une position dominante, à celle de subalterne, puis à nouveau à une position de domination relative, à un moment où les structures politiques de cette région du golfe de Guinée vont s'effondrer pour laisser place à l'ordre colonial: le personnage central du roman officiait comme maître des rituels à la cour du roi d'Abomey quand il est trahi puis déporté au Brésil avant de revenir à Agoué, non loin de Ouidah, à quelques kilomètres du port où il embarqua. Au cours de ce périple, il apprendra plusieurs langues et changera plusieurs fois de nom, de métier, de religion et d'état d'âme.

A partir de la rencontre avec l'auteur ${ }^{5} \mathrm{du}$ roman historique mentionné, a succédé une série de rencontres donnant lieu à des 
discussions qui prirent progressivement la tournure de la préparation d'une pièce de théâtre, en raison de la préoccupation professionnelle commune de ce réseau, au fur et à mesure que s'esquissait le projet d'une adaptation à la scène du roman.

Si suivant l'exemple de Johannes Fabian, il fut initialement question de développer cette enquête ethno-théâtrale dans le cadre d'une compagnie de théâtre loméenne existante, cette possibilité disparut toutefois avec la faillite de la structure pressentie, se cumulant aux difficultés financières rencontrées par le chercheur, ralentissant sa progression.

C'est toutefois grâce à l'implication artistique des anciens membres de cette compagnie, notamment de l'écrivain Kangni Alem, du metteur en scène Gaétan Noussouglo et de l'acteur Léo Ayihivi, que le projet ne fut jamais complètement abandonné et que les discussions purent se poursuivre jusqu'à aujourd'hui. A cette équipe s'est ensuite rapidement ajouté Azé Kokovivina, le maître du Concert-Party, un genre de théâtre populaire en vogue à Lomé.

Désormais dans un cadre informel, sans affiliation institutionnelle unique, et motivés par la perspective d'une création théâtrale dont nous serions les partenaires, nos contributions allaient se développer dans l'esprit d'une franche camaraderie et d'une amitié de longue date. Il est vrai que le temps de l'enquête - et sans doute aussi au-delà - le terrain provoque des échanges qui établissent un ensemble de relations dont l'esthétique devrait se retrouver dans la restitution qui en est faite, alors que trop souvent le texte produit est décharné de tout corps, vidé de l'expérience qui l'a pourtant rendu possible.

Autour de ce noyau fondateur se sont ensuite mis à graviter diverses personnes, s'inscrivant dans la démarche de manière différente, avec un investissement et une présence chronologique variables.

Il apparaît ainsi que l'objet d'une recherche anthropologique est donc d'abord composé de personnes dont le statut oscille entre celui de collaborateur, d'informateur et d'ami; la relation qui peut en être faite doit donc être à la hauteur de ce rapprochement, au nom de l'amitié. Une fois que les discussions préalables eurent permis de définir un certain cadre de discussion, il fut possible de réaliser entre 2009 et 2014 - et grâce au soutien d'institutions diverses (Institut français de Lomé, le laboratoire IRIS - Institut de Recherche Interdisciplinaire sur les enjeux sociaux et des contributions privées) -4 
séjours au Togo d'une durée moyenne de 14 jours chacun au cours desquels s'est déroulé un premier volet de la collecte de récits autour de la mémoire brésilienne.

En aucune manière, la recherche qui se trouve esquissée ici se limiterait à ces séjours. Ce terrain multi-site se confond avec la construction de l'objet dont les moments ne pourraient se réduire à une localisation géographique, en suivant le déplacement physique des participants, entre le Togo, la France et le Brésil. Quantité d'échanges se sont aussi faits par le biais d'Internet: soit par mail soit par les réseaux sociaux du type Facebook.

\section{Du Terrain à la Scène: de la description ethnographique à la dramaturgie}

Dès lors, une fois entériné le principe d'une enquête ethnographique qui prend la forme d'une création théâtrale, il nous faudra formuler un texte de travail, littéralement un canevas ${ }^{6}$, sorte de synopsis ou pré-texte dramaturgique procédant de l'enquête ethnographique, à la fois à partir du contenu des récits collectés et de l'analyse des enjeux sociaux de la situation étudiée, qu'il s'agit d'amener/transposer sur/à la scène.

Pour l'heure, il s'agit de fixer les récits collectés en les ordonnant dans une structure narrative et épique qui reprend le périple emblématique de la personne capturée, devenue esclave au Brésil et qui en revient affranchie pour occuper une position dominante dans la nouvelle société émergeante, à la veille de la colonisation.

De manière à rester fidèle à l'ambiguïté qui entoure l'identité brésilienne, telle qu'elle ressort des entretiens, il faudra par la dramaturgie décrire ce jeu d'identités dont témoigne la biographie des Afro-Brésiliens, qu'il s'agisse de personnes ayant existé ou de personnages fictionnels, de descendants directs des revenus ou de ceux qui s'en réclament. Ce faisant, on s'intéressera à décrire un jeu de masques hétéroclite, plutôt qu'une structure culturelle homogène.

Cette malléabilité culturelle, cette aptitude à être plusieurs personnes en même temps ou au cours d'une existence, semble en réalité constituer notre objet. Ainsi, plutôt que de tenter de saisir les caractéristiques d'une culture, il s'agit ici de rendre compte de l'art des hommes à appartenir à plusieurs cultures, s'inscrivant ainsi dans une anthropologie véritable, une science de l'homme et non plus une 
science des ethnies. Dès lors, la description ethnographique devient essentiellement dramaturgique. Elle débarrasse le chercheur de son boulet d'herméneute, au profit d'une description minimale, c'està-dire anthropologique (universelle), rejoignant sur ce point assez clairement le projet d'une praxéographie annoncé par Jean Bazin quand il suggère que "[...] la compréhension s'obtient par un travail de généralisation, c'est-à-dire de réduction de l'altérité apparente, qu'il faudrait appeler praxéographie, plutôt qu'ethnographie, si le terme n'était pas d'allure aussi pédant" (Bazin, 2000). La proposition inverse le menant logiquement à constater que "moins je sais décrire, plus je suis tenté d'interpréter” (Bazin, 2008, p. 417).

De manière apparemment paradoxale, l'on n'essaiera donc pas de montrer ce qu'est l'identité brésilienne, mais en quoi le fait qu'elle ne soit pas saisissable, isolable ou essentialisable, permette justement de la qualifier, faisant de cette complexité l'indice d'une histoire composite, ballotée dans l'Atlantique, et enchevêtrée dans l'histoire globale. C'est bien de cette histoire multiple dont nous parlent d'abord les habitants des sociétés côtières du Togo quand on leur demande de formuler ce que leur évoque le Brésil. Dans cette perspective, l'expérience du terrain nous oblige à envisager l'hétérogénéité culturelle et sociale des sociétés de l'ancienne Côte des Esclaves comme fondatrice de son identité. En conséquence, il nous faut radicalement évacuer la recherche d'une pureté culturelle originelle.

\section{La Fabel Brechtienne et la Connaissance Anthropologique}

Dans une perspective d'écriture théâtrale, il s'agit dès lors de faire émerger à partir des récits collectés un noyau dramatique, une Fabel (Brecht, 1961). La Fabel - à ne pas confondre avec la fable - est un terme technique propre à la dramaturgie brechtienne que l'on ne retrouve pas dans toutes les démarches théâtrales et désigne l'enjeu social d'une pièce de théâtre. La Fabel devra être formulée de manière à donner aux acteurs et au metteur en scène de la pièce des indications de jeu permettant une mise en situation historique. La Fabel met en lumière les relations de pouvoir en présence, de manière à en traduire le sens, sans en reproduire l'effet, par une image distanciée, permettant au spectateur d'accéder à une lecture critique.

De la scène au terrain, la Fabel théâtrale pourrait de plus désigner la problématique anthropologique, provoquant ainsi une rencontre 
insolite entre deux disciplines, la dramaturgie de la pièce et la description ethnographique se rejoignant au point de se confondre. Ainsi, il s'agit de qualifier la situation sociale qui sous-tend la pièce dans un cas ou de désigner l'objet de la recherche anthropologique dans l'autre cas; on s'intéressera aux leviers économiques de la situation à restituer (que ce soit sur scène ou dans l'analyse ethnographique proposée), l'ensemble étant restitué dans une démarche didactique, par une description suffisamment ouverte pour que tout acteur, quelle que soit son origine, puisse comprendre les ressorts de son rôle dans une action théâtrale donnée.

Il s'agit d'introduire un effet de distanciation, tel que défini par Brecht en tant que procédé utilisé dans le cadre d'une narration fictionnelle, ayant pour objectif d'interrompre le processus naturel d'identification du lecteur ou du spectateur aux personnages auxquels il est confronté. La distanciation a pour objectif de produire l'effet inverse quand elle est destinée au public. En effet, lorsqu'il est interdit au public, même brièvement, de s'identifier au personnage, l'effet produit sera de lui faire prendre ses distances, cette fois par rapport à la fiction, en le renvoyant à sa condition de spectateur ou de lecteur. Ce procédé peut être utilisé dans toute forme de narration, tant au théâtre qu'en littérature, à la télévision ou au cinéma (et en anthropologie?).

Du théâtre brechtien à la méthode praxéographique, de Brecht à Bazin et de la distanciation à la réflexivité critique, l'intrusion du chercheur sur le terrain est évoquée comme ayant permis une prise de conscience aux acteurs sociaux, faisant émerger une réalité que l'engagement dans l'action dissimulait. Cette prise de conscience, ou réalisation, des participants d'une situation sociale, alors que le chercheur progresse dans la construction de son objet, est comparable au travail que réalise de son côté le dramaturge quand il identifie dans un texte théâtral les ressorts sociaux et les implications politiques de telle action théâtrale.

Les étapes de la fabrication de la pièce de théâtre deviennent ainsi un terrain, composé d'ateliers qui génèrent des échanges permettant la construction de l'objet. L'ethnologue est ainsi invité à endosser la fonction du dramaturge - tel que Bertolt Brecht défini ce métier - en collaborant activement à construction de son objet et à l'élaboration de l'œuvre (c'est-à-dire à la création d'un spectacle). 
Cette coopération se situe sur plusieurs plans: si elle permet l'intégration d'informations ethnographiques, elle tend aussi à intervenir sur le processus créatif de la pièce, en proposant une dramaturgie, c'est-à-dire la restitution de la structure des relations qui relient les personnages les uns aux autres, en écho à la façon dont un passé - en l'occurrence la mémoire brésilienne - est aujourd'hui réactualisé en fonction d'enjeux contemporains.

De manière à relater l'ambiguïté fondamentale de l'identité brésilienne, telle qu'elle ressort des discussions, il faudra par la dramaturgie, décrire ce jeu d'identités dont témoigne la biographie des Afro-Brésiliens qu'il s'agisse de personnes ayant existé ou de personnages fictionnels, de descendants directs des revenus ou de ceux qui s'en réclament. Ainsi, plutôt que de tenter de saisir les caractéristiques d'une culture, il s'agit ici de rendre compte de l'aptitude qu'ont les hommes à s'inscrire dans plusieurs registres culturels et à se transformer, faisant ainsi du changement le véritable objet de l'anthropologie.

C'est à cette réflexion que nous invite les échanges autour du théâtre. Ce genre est-il autre chose qu'une méditation enjouée sur l'identité? S'il fallait définir l'anthropologie que j'ambitionne, ne pourrait-on pas aussi la décrire comme une science de la transformation et de la diversité, saisissant en l'homme son aptitude au changement, sa capacité à devenir autre. Cette analyse nous éloigne définitivement de la conception classique de l'ethnologie, dont la dimension monographique apparaît par exemple dans la définition proposée par le petit Larousse: "Étude descriptive des activités d'un groupe humain déterminé (techniques matérielles, organisation sociale, croyances religieuses, mode de transmission des instruments de travail, d'exploitation du sol, structures de la parenté)" (Larousse, 2014).

Inversement, il apparaît que le déguisement, la transformation, l'hétérogénéité ou la multiplicité sont des caractéristiques plus constantes de l'humanité que la statique culturelle, ou la structure d'un groupe humain déterminé qui, à peine identifiée, semble aussitôt affadie, se teintant déjà des autres couleurs de l'histoire. Ainsi, si l'ethnologie envisage sa tâche comme une mise en lumière des systèmes culturels, l'anthropologie envisagée ici se contentera de se définir comme un commentaire de processus humains, en situation, 
dans le changement. Ce constat autorise à penser que l'étude de ce qui est théâtral en l'homme relève de l'anthropologie véritable, visant à comprendre l'homme dans la variété de ses histoires.

Loin de rejeter le statut de savoir des productions anthropologiques, il s'agit ici d'en souligner la spécificité. Il apparaît que ce qu'il faut savoir pour composer une pièce de théâtre, du moins dans une logique brechtienne, rejoint une forme de connaissance anthropologique, faisant ainsi du théâtre une sorte d'arène anthropologique, se combinant théoriquement dans un même principe ethno dramaturgique ${ }^{7}$.

En passant de l'observation à la communication, de l'information à la conversation, par la mise en place - autour d'une enquête théâtrale - d'un espace de discussion autour d'une problématique de recherche partagée, la nature du savoir produit tend à se modifier. Si d'un côté il perd de sa pertinence informative, il gagne en acuité relationnelle. Ce glissement permet précisément de qualifier le savoir ainsi produit de dramaturgique (éventuellement d'ethno dramaturgique), c'est-à-dire qu'il devient indissociable du contexte dans lequel il s'énonce au point même de rendre complexe sa restitution en dehors de son cadre de production. Cette propriété quelque peu aporétique du savoir anthropologique ainsi défini en fait un genre à part, la rapprochant d'un exercice littéraire (en l'occurrence théâtral) sans toutefois lui faire abandonner son ambition scientifique, pardelà la science et l'art.

Le cadre donné par le processus de création, ainsi que l'attention portée à l'histoire brésilienne de cette partie de la côte africaine (à moins que ce soit l'histoire africaine du Brésil, ou encore l'une et l'autre), est un dispositif interactif qui procède de l'activation d'un champ symbolique dont les contours ne recoupent pas ceux d'une éventuelle culture ou d'un quelconque système de représentation qui ne tiendrait sa cohérence que de lui-même. Dans l'espace qui est un temps animé par la curiosité anthropologique, l'objet de la recherche devient un thème de discussion collectif. Il fait écho à la multiplicité des dimensions de cet objet complexe dont la construction sous forme de recherche, dans un terrain, implique le recours permanent à une multitude de registres, qu'il s'agisse de la langue, de la vie économique ou encore de la religion, dans des sociétés profondément hybrides. 


\section{La Lettre Oubliée}

Partant d'un canevas simple (le récit universel d'une personne qui part et qui revient, comme synthétisé dans le roman Esclaves de Kangni Alem) on présentera une série de tableaux qui seront alimentés des récits collectés au sud du Togo, autant auprès des descendants des Afro-Brésiliens que des autres habitants de la région. On y intégrera aussi les récentes productions littéraires et études scientifiques portant sur l'histoire des communautés brésiliennes et de leur actualité. Dans un second temps (dont il ne sera pas rendu compte ici), ces tableaux seront finalisés au cours d'une résidence d'écriture.

Pour l'instant, le tableau initial de la pièce, qui en contient aussi la fable, est inspiré du récit fondateur de la famille D'Almeida d'Aného tel qu'il nous a été raconté à plusieurs reprises et encore tout récemment, en mai 2014. Proche de la famille de Souza, Apetoh Ayi, fondateur de la lignée, est le fils de la sœur de la deuxième épouse de Francisco Félix de Souza, princesse de Glidji. Ayi, dont la mère meurt en couches, est recueilli par Chacha. Il vit dans la maison, sans bénéficier de la même éducation que les enfants de Souza. Francisco Felix de Souza découvre alors l'intelligence du garçon, l'adopte et lui donne le nom de Pedro Felix Ayi d'Almeida, du nom de son ami portugais d'Almeida, mort sans avoir laissé de descendance. Le chef de la famille D’Almeida me raconte (entretien du 7 juin 2014, Lomé) un détail qui se dit encore dans la famille, et qu'il me confie aussi avoir transmis à ses enfants: il semblerait que le petit Apetoh Ayi avait pour tâche domestique d'accompagner à l'école (nous sommes vers 1845 , et il n'y a qu'à l'église qu'il est possible d'être alphabétisé, et en portugais) les enfants de sa tante, qui eux avaient le droit d'apprendre à lire et à écrire. Un jour, une lettre cachetée adressée à la mère, arriva du Brésil. Le contenu en semblait important. Ne voulant risquer d'exciter la convoitise de son entourage, la mère des neveux du petit Apetoh Ayi demanda à ses enfants de lire la lettre, ce à quoi ils échouèrent. A la grande surprise de tous, c'est Apetoh Ayi qui parvint à déchiffrer la lettre, et à la traduire en langue mina. L'actuel chef de la famille D'Almeida qui me raconte cette histoire dans son bureau climatisé d'homme d'affaire, situé dans le centre historique de la ville de Lomé, insiste: "il a appris le portugais en écoutant aux portes de l'école où il accompagnait ses neveux, et rajoute qu'eux n’ont rien appris, mais lui si”. 
Etrangement, personne ne se souvient du contenu de la lettre.

Quel est son contenu?

La réponse à cette question sera laissée en suspens et donnera le fil directeur aux prochains ateliers, envisagés dans les mois à venir, jusqu'à la création en septembre 2016 d'une pièce de théâtre.

\section{Conclusion}

Comme esquissé dans cet article qui traite, rappelons-le, d'une enquête théâtrale en cours, le chercheur en vient à occuper une place de dramaturge dans un processus collectif qui évolue en permanence à moteur ouvert - c'est-à-dire dans une démarche où les étapes de la fabrique du savoir autant que de l'œuvre sont visibles - et au cours duquel la problématique ne cesse d'évoluer. L'intervention des participants influence la construction de l'objet, le terrain ne pouvant plus en aucune manière être considéré comme un mode de vérification scientifique, comme une situation d'enquête ayant son histoire et sa réalité propre.

Dans une démarche expérimentale à la croisée du théâtre et de l'anthropologie, il s'agit de mettre à l'épreuve une méthode ethnodramaturgique, c'est-à-dire une approche conçue de manière à se servir du théâtre comme d'un moyen permettant de provoquer une situation d'enquête, les étapes de la réalisation d'une pièce de théâtre, depuis les recherches préalables jusqu'aux répétitions, qu'il s'agisse du texte, des décors, des costumes ou de la musique se superposant au développement d'une enquête ethnographique.

Ainsi, plutôt que de considérer que le chercheur en anthropologie doive agir en se faisant l'observateur le plus discret possible d'une réalité qu'il n'est pas sensée affecter, on se propose ici de produire la situation sociale à étudier. On rompt ainsi avec la précaution de non-contamination qui régit en général les règles positives de l'observation ethnographique. Ce faisant, il s'agit de créer un dispositif qui devrait permettre à l'ensemble des personnes impliquées dans la recherche, chercheurs autant qu'enquêtés, de participer à celle-ci de manière opératoire, c'est-à-dire en en orientant le cours.

Ce choix méthodologique a des implications théoriques considérables. Il résulte d'un refus de perpétuer les relations de pouvoirs qui sous-tendent la relation d'enquête, la connaissance ne pouvant être 
envisagée comme le résultat d'une lutte de pouvoir. En provoquant une situation d'enquête, en mettant le chercheur et les enquêtés en coprésence, on espère idéalement que la construction de l'objet de la recherche se fasse de manière coopérative et dialogique, réduisant ainsi l'asymétrie habituelle.

Singulièrement, il apparaît que la modification des conditions de production de l'enquête ethnographique transforme aussi le statut épistémologique du savoir ainsi produit sur la communauté étudiée. En passant d'une structure informative à une situation dialogique, on transite d'un paradigme à un autre. Au cours de cette mutation le savoir gagne en densité relationnelle et descriptive, mais perd en objectivité. De plus en plus difficile à extraire du contexte dans lequel il est produit, l'enquête devient sa propre archive, rendant complexe sa restitution.

Ce faisant, en jouant avec les limites convenues des disciplines, il s'agit de chercher une posture de recherche recevable sur le plan éthique, impliquant les personnes sur qui porte l'enquête dans la construction de l'objet, dans un souci de collégialité, voire de démocratie participative.

Proche de mes convictions morales, un terrain doit donc permettre - du moins de le tenter - de se dégager des rapports de pouvoir. En rappelant que, dans l'idéal, le terrain doit donc être un environnement, une écologie politique, donnant les clés de libération pour l'ensemble de ceux qui s'y trouvent engagés, informateurs et informés, enquêteurs et enquêtés confondus. Dans cet engagement radical et utopique, ne devrions-nous aussi exiger de l'anthropologie ce qu'André Gorz attend de la philosophie: "Je ne comprends donc pas la philosophie à la manière des créateurs de grands systèmes philosophiques, mais comme la tentative de se comprendre, de se découvrir, de se libérer, de se créer" (Duverger, 2013, n.p.)? 


\section{Notes}

${ }^{1}$ Cette contribution s'inscrit dans le prolongement de l'article Le terrain: un théatre anthropologique (Müller, 2013).

${ }^{2}$ Il s'agit d'une recherche en cours qui a reçu le nom de projet Zomayi (2013).

${ }^{3}$ Un terreiro de candomblé est le terme généralement utilisé pour désigner les lieux de culte du candomblé qui est en même temps un espace culturel communautaire.

${ }^{4}$ Cette représentation théâtrale dansée est l'une des expressions culturelles les plus diffusées dans le Brésil, après le carnaval. L'origine du bumba-meu-boi remonte au XVIII siècle, résultant de la relation inégale entre esclaves et seigneurs, il reflète les conditions sociales vécues par les noirs et les indiens.

${ }^{5}$ Celui-ci ressent non sans ironie, comme pour rappeler l'ambition artistique et non scientifique de son projet, le besoin de rappeler en exergue de son livre que "[...] l'impulsion qui est à l'origine [de ce livre] étant de nature esthétique plutôt qu'érudite, j'ai exploité les décors de l'Histoire non tant avec l'arrière-pensée de mettre en scène plus ou moins fidèlement des événements qui se trouvent consignés dans les livres que mû par ce qu'ils m'apportent de plaisir et d'émerveillement. C'est de propos délibéré que joué de l'anachronisme, que j'ai inventé une langue, des mots, bref, que j'ai laissé mon inspiration divaguer bien au-delà de mes sources premières. Chaque fois que la vérité historique allait à l'encontre de l'invention créatrice, je l'ai remodelée, en pleine et lucide connaissance de cause, afin de satisfaire les exigences de mon propos" (Boyle, 1993, p. 359).

${ }^{6}$ Le canevas est le récit détaillé de l'histoire que l'on veut écrire sous la forme d'une pièce de théâtre. Il renferme toute la structure de la pièce. Il se divise en scènes, et chacune des scènes se divise en actions.

7 "Ce qu'il nous est possible de savoir ou d'apprendre à propos d'une culture/société n'apparaît pas sous forme de réponses à nos questions, mais comme performance dans laquelle l'ethnologue agit, comme Victor Turner l'a formulé un jour, à la manière d'un ethno dramaturge, c'est-à-dire comme quelqu'un qui cherche à créer des occasions au cours desquelles se produisent des échanges significatifs" (Fabian, 1999, p. 124).

\section{Références}

ALEM, Kangni. Esclaves. Paris: Lattès, 2009.

AMOS, Alcione M.; AYESU, Ebenezer. "I am Brazilian”, History of the Tabon, Afro-Brazilians in Accra, Ghana. Transactions of the Historical Society of Ghana, Accra, n. 6, p. 35-58, 2002.

BAZIN, Jean. Des Clous dans la Joconde. Toulouse: Edition Anarcharsis, 2008.

BAZIN, Jean. Université de tous les Savoirs. Conference du 5 avril 2000. France, 2000. Disponible sur: <http://www.canal-u.tv/producteurs/universite_de_tous_les_savoirs/dossier_programmes/les_conferences_de_l_annee_2000/deuxieme_etape_de_reflexion_et_ de_critique_y_a_t_il_encore_des_sciences_humaines/l_anthropologie_en_question_alterite_ou_difference>. Consulté le: 09 avril 2014.

BOYLE, T. Coraghessan. Water Music. London: Granta Books, 1993. 
BRECHT, Bertolt. Kleines Organon für das Theater. Berlin: Suhrkamp, 1961.

CLIFFORD, James (avec George Marcus). Writing Culture: the poetics and politics of ethnography. Los Angeles: University of California Press, 1986.

DUVERGER, Timothée. Écologie et autogestion dans les années 1970. Discours croisés d'André Gorz et de Cornelius Castoriadis. Ecologie \& Politique, Paris, Presses de Sciences Po (P.F.N.S.P.), n. 46, p. 140-144, 2013.

FABIAN, Johannes. Power and Performance - Ethnographic Explorations through Proverbial Wisdom and Theatre in Shaba, Zaïre. Madison: University of Wisconsin Press, 1990.

FABIAN, Johannes. Theater and Anthropology, Theatricality and Culture. Research in African Literatures, Cambridge, Cambridge University Press, v. 30, n. 4, p. 24-31, Winter 1999.

GURAN, Milton. Les “Brésiliens” du Bénin. Paris: Editions La Dispute, 2010.

HUBER, Magnus. The Nova Scotia-Sierra Leone connection. New evidence on an early variety of African American Vernacular English in the diaspora. In: ESCURE, Geneviève; SCHWEGLER, Armin (Ed.). Creoles, Contact, and Language Change. Linguistic and social implications. Amsterdam: Benjamins, 2004. P. 67-95.

LAROUSSE, Le petit dictionnaire. Paris: Larousse, 2014.

LAW, Robin. Ouidah: The Social History of a West African Slaving 'Port', 1727-1892. Oxford: James Currey, 2004.

MÜLLER, Bernard. Le Terrain: un théâtre anthropologique. Revue Communications, Paris, Seuil, n. 92, p. 75-82, juillet 2013.

SOUMONNI, Elisée. The Afro-Brazilian Communities of Ouidah and Lagos: a Comparative Analysis. In: CURTO, Jose C.; SOULODRE-LA FRANCE, Renee. Africa and the Americas: interconnections during the Slave Trade. Trenton: Africa World Press, 2005. P. 12-15.

ZOMAYI - CABARET ATLANTIQUE. Projet Zomayi (site internet). France, 2013.

Bernard Müller est anthropologue et dramaturge. Il étudie les processus de mise en scène qu'il s'agisse de dispositifs scéniques (théâtre, rituels, performance, etc.), de scénographies muséales ou de toute situation qui relève explicitement de la performance. Depuis 2003, il dirige le séminaire "mise en scène et en récit" à l'Ecole des Hautes Etudes en Sciences Sociales (Paris) où il est membre de l'Institut de Recherche Interdisciplinaire sur les enjeux sociaux (IRIS). Il coordonne également une plateforme de recherche sur le spectacle, envisagé comme un collectif transdisciplinaire sur un mode collaboratif et dans une perspective internationale (www.curioweb.org).

E-mail:muller@ehess.fr

Ce texte inédit, révisé par Annelyse Gayraud, est également publié en portugais dans ce numéro.

Reçu le 23 avril 2015

Accepté le 31 août 2015 